\title{
Inferior calcar buttress reduction pattern for displaced femoral neck fractures in young adults: a preliminary report and an effective alternative
}

\author{
Wen-Feng Xiong ${ }^{1}$, Shi-Min Chang ${ }^{1 *}$, Ying-Qi Zhang ${ }^{2}$, Sun-Jun Hu ${ }^{1}$ and Shou-Chao Du ${ }^{1}$
}

\begin{abstract}
Background: Fracture reduction quality is of paramount importance for osteosynthesis. The aim of this study was to report the outcome of an inferior cortical buttress non-anatomic reduction pattern and internal fixation for displaced femoral neck fractures (Garden types III and IV) in young adults.

Methods: A retrospective analysis of 46 displaced femoral neck fractures was performed, which were treated by closed reduction and internal fixation with parallel cannulated screws. There were 20 males and 26 females, with an average age of 50.3 years (19-60). According to the inferior cortical reduction quality seen in recorded intraoperative fluoroscopy, the patients were divided into two groups. Group I $(n=30)$ was anatomic cortical apposition as the two inferior cortices were smoothly contacted, and group II $(n=16)$ was buttress cortical apposition as the two inferior cortices were located in positive support contact (Gotfried reduction pattern). With a mean follow-up of 22.0 months, femoral neck length, neck-shaft angle, and clinical outcomes were compared.

Results: Thirty-nine patients (84.8\%) achieved uneventful fracture union. Complications occurred in seven patients, six in group I (20\%) and one in group II (6.3\%), including displacement to varus, neck shortening, early fixation failure, nonunion, and avascular necrosis of the femoral head. No significant difference existed in the complication rate between the two groups $(p=0.216)$. Four patients $(13.3 \%)$ in group I were converted to prosthetic replacement, but none in group II.

Conclusions: For closed reduction and fixation of displaced femoral neck fractures in young adults, an inferior cortical buttress reduction pattern, though non-anatomic, can produce sustainable fracture stability and predictable clinical outcomes.
\end{abstract}

Keywords: Displaced femoral neck fractures, Inferior cortical buttress reduction, Gotfried non-anatomic reduction, Calcar support, Multiple cannulated screws

\section{Background}

Femoral neck fractures in young adults usually result from high-energy trauma [1]. These injuries may involve a displaced fracture pattern, resulting in instability of the fracture site, and this may be associated with complications including fixation failure, neck shortening,

\footnotetext{
* Correspondence: shiminchang11@aliyun.com

${ }^{1}$ The Department of Orthopaedic Surgery, Yangpu Hospital, Tongji University School of Medicine, 450Tengyue Road, Shanghai 200090, People's Republic of China

Full list of author information is available at the end of the article
}

nonunion, or head avascular necrosis of the femoral head $[2,3]$.

Besides the degree of initial fracture injury, anatomic reduction is thought to be the key for successful uneventful fracture union. There is no doubt that excellent reduction quality is the most important procedure for the treatment of displaced femoral neck fracture, before any kind of internal fixation is carried out. However, a true exact anatomic reduction may be difficult to justify in closed reduction and fluoroscopy monitoring. In 2012, Gotfried et al. [4] proposed a non-anatomic 

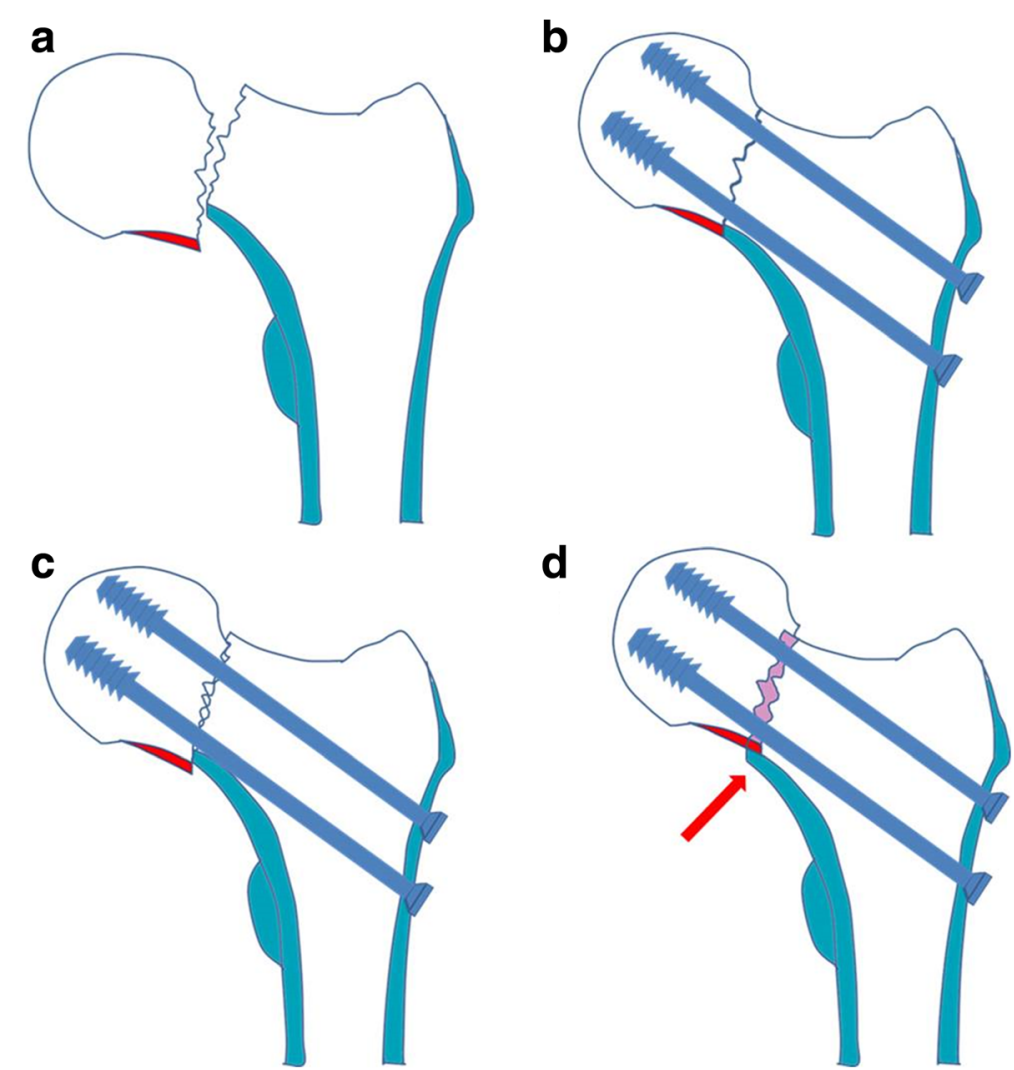

Fig. 1 Schematic drawing to show the inferior cortical apposition pattern after manual reduction in displaced femoral neck fractures. a The displaced femoral neck fracture. $\mathbf{b}$ Anatomic cortical apposition with smooth inferior cortex apposition. $\mathbf{c}$ Negative cortical apposition lost calcar support. d Positive cortical apposition obtained calcar support. The proximal head-neck fragment is slightly superiorly displaced intentionally (less than one cortex thickness or $4 \mathrm{~mm}$ ) to the distal fragment (red arrow), so that the inferior cortex of the proximal head-neck fragment (red color) can be buttressed by the calcar cortex of the distal neck fragment (blue color). After screw fixation, the femoral head-neck is dual supported by the fixation screw and the calcar cortex, and a sustainable stability is restored

cortical buttress reduction pattern with inferior calcar support (Fig. 1) and obtained favorable clinical results in a small number of patients [5]. However, no other report till now was presented in the literature.

We retrospectively reviewed our patient data and attempted to evaluate the value of this new cortical buttress reduction pattern and compare the results with the anatomic reduction.

\section{Materials and methods \\ Patients}

After Institutional Review Board approval (No. 2017ZRKX-013), we retrospectively reviewed our data from January 2014 to January 2017. The inclusion criteria were as follows: (1) 60 years of age or less, (2) isolated and displaced fractures (Garden types III and IV), (3) closed reduction and fixation with multiple cannulated screws $(6.5 \mathrm{~mm}$ in diameter) in inverted triangle or diamond configuration, and (4) more than 1 year of follow-up. Patients with a pathological fracture and a malignant disease, open fracture, previous fracture, symptomatic osteoarthritis of the hip before injury, and rheumatoid arthritis, as well as those without complete sets of preoperative, postoperative, and follow-up radiographs, were excluded.

There were 46 patients who met the criteria and were included in this study. The subject group was comprised of 20 males and 26 females, with an average age of 50.3 years (range, 19-60 years). Personal age at the time of surgery, body mass index (BMI), score of American Society of Anesthesiologists (ASA), and follow-up period were recorded retrospectively after patient inclusion. All information was recorded, and all evaluations were performed by the senior visiting orthopedic staff.

\section{Fracture management}

The patients were positioned on a fracture table, and fluoroscopy with an image intensifier was used routinely to guarantee the quality of the procedure. As a general rule in practice, firstly, we performed closed manual reduction and evaluated the fracture reduction quality by fluoroscopy. Besides Garden alignment (residual angle) 
in AP and lateral views, we accepted two cortical apposition patterns: anatomic inferior smooth reduction (Fig. 2) and non-anatomic inferior buttress reduction (Figs. 3 and 4). If closed reduction was not satisfied after two attempts, open reduction through Watson-Jones approach was carried out and those patients were excluded from this study.

Postoperatively, sitting was encouraged at the first day, non-weight-bearing ambulation with the aid of a walker frame was allowed after 1 or 2 weeks as long as the pain was tolerated. Patients were encouraged to partial weight bearing 6 weeks after the operation. For patients in whom radiographic and clinical healing appeared to be progressing towards union at 6 weeks, weight bearing was advanced slowly from toe touch to partial weight bearing with a walker frame over the subsequent 6 weeks. When the clinical and radiological signs of union were presented, full weight bearing was allowed.

The patients were routinely followed up at 6 weeks, 3 months, 6 months, and 12 months postoperatively and annually thereafter, and follow-up plain radiographs were obtained at every follow-up visit. In addition, as postoperative lateral radiography often did not meet the standard criteria, some patients were further evaluated with CT scanning.

\section{Radiographic assessment}

The quality of fracture reduction was evaluated using the recorded images of intraoperative fluoroscopy. All enrolled patients were divided into two groups: those reduced with anatomic reduction (group I) and those with non-anatomic cortical buttress reduction (group II).

Garden alignment index angles on intraoperative fluoroscopy (both AP and lateral views) were measured to elevate the degree of residual angulation. Two parameters were calculated to detect possible changes on intraoperative fluoroscopy and postoperative follow-up radiographs. The femoral neck-shaft angle (FNSA), which is the angle between the two axes of head-neck and shaft medullary, was measured. Changes of more than $10^{\circ}$ were defined as displacement to varus. Femoral neck length (FNL) was measured according to the method introduced by Zlowodzki et al. [6], and more than 5-mm changes were defined as shortening.

Early failure was defined as loss of reduction requiring a reoperation within 6 months. Nonunion was

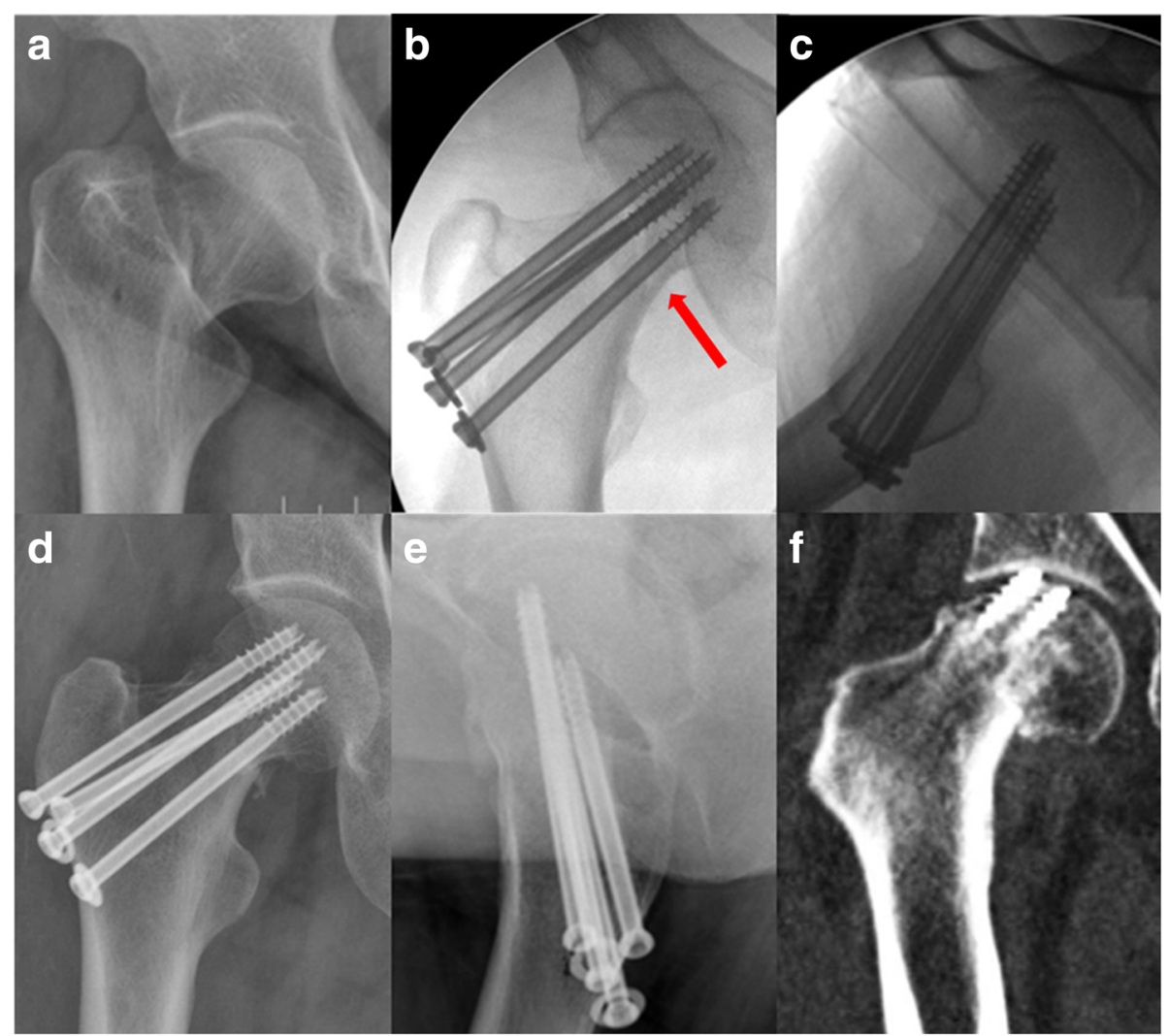

Fig. 2 AP view of a displaced femoral neck fracture in a 55-year-old female (a). The fracture obtained smooth anatomic reduction (red arrow) according to intraoperative fluoroscopies $(\mathbf{b}, \mathbf{c})$, and the fracture changed into slightly varus $\left(\leq 10^{\circ}\right) 3$ days postoperatively $(\mathbf{d}$, e). CT showed the fracture displaced to varus $\left(>10^{\circ}\right) 4$ months postoperatively, which resulted in early failure as the screws cut out from the femoral head (f) 


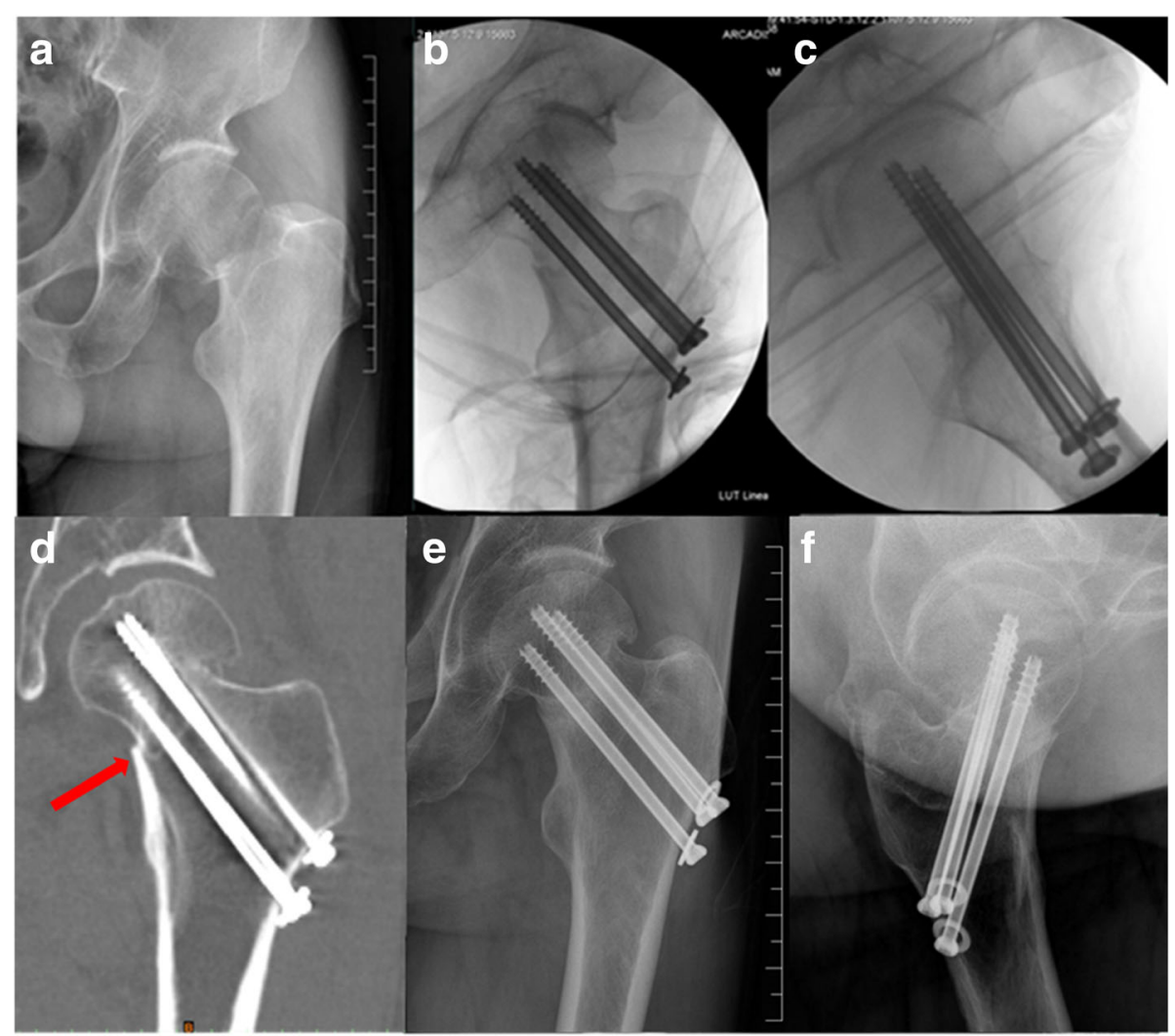

Fig. 3 (a) A 56-year-old male suffered from displaced femoral neck fracture. (b, c) Intraoperative fluoroscopies showed that inferior cortical buttress pattern reduction was obtained. (d) CT scanning demonstrated the inferior medial cortices were resisted on a positive position (red arrow). (e, f) In six months follow-up, the fracture healed

defined as a clearly visible fracture line at 12 months postoperatively.

\section{Statistical analysis}

All statistical calculations were performed using SPSS version 19.0 (SPSS Inc. Chicago, IL, USA). Basic descriptive statistical analyses were used to describe the patient population and treatment outcomes. The MannWhitney $U$ test was used for continuous data. The $\chi^{2}$ test or Fisher exact test was used for categorical variables. Statistical significance was defined as $p<0.05$.

\section{Results}

For the group as a whole, the mean follow-up time was 22.0 months (range, 12-36 months). No statistical difference in gender, age, body mass index (BMI), American Society of Anesthesiologists (ASA) score, and follow-up time was observed between the two groups (Table 1).

There were 30 cases classified as cortical anatomical reduction, and 16 cases as cortical buttress reduction. The Garden alignment index angles were $165.1^{\circ}$ in average in AP view and $178.2^{\circ}$ in lateral view. No statistical difference was revealed between the two groups $(p=0.104)$ by the Mann-Whitney $U$ test. During follow-up, 39 patients (84.8\%) achieved fracture union uneventfully. Complications occurred in seven patients, six in group I (20\%) and one in group II (6.3\%). No significant difference existed in the complication rate between the two groups $(p=0.216)$. Four patients $(13.3 \%$, all in group I) were converted to prosthetic replacement. However, the Fisher exact test revealed no significant difference between the two groups $(p=0.168)$ (Table 2).

The mean varus change of the femoral neck-shaft angle was $3.4^{\circ}$ (Table 3 ). Considering varus displacement in postoperative follow-up, no significant difference was noted between the two groups $(p=0.715)$. Using $10^{\circ}$ as a cutoff criterion, three fractures (10\%) displaced to varus in group I. In a female patient, the anatomic reduction changed into slightly varus $\left(\leq 10^{\circ}\right)$ in 3 days and gradually had a screw cutout in 4 months (Fig. 2). The patient accepted conversion to prosthesis. The remaining two gradually displaced to varus, both of them were diagnosed with fracture nonunion 6 months postoperatively and accepted conversion to total hip arthroplasty (THA). In group II, one patient gradually displaced to varus and had femoral neck shortening, but the fracture was eventually united without discomfort in 6 months (Fig. 4). 


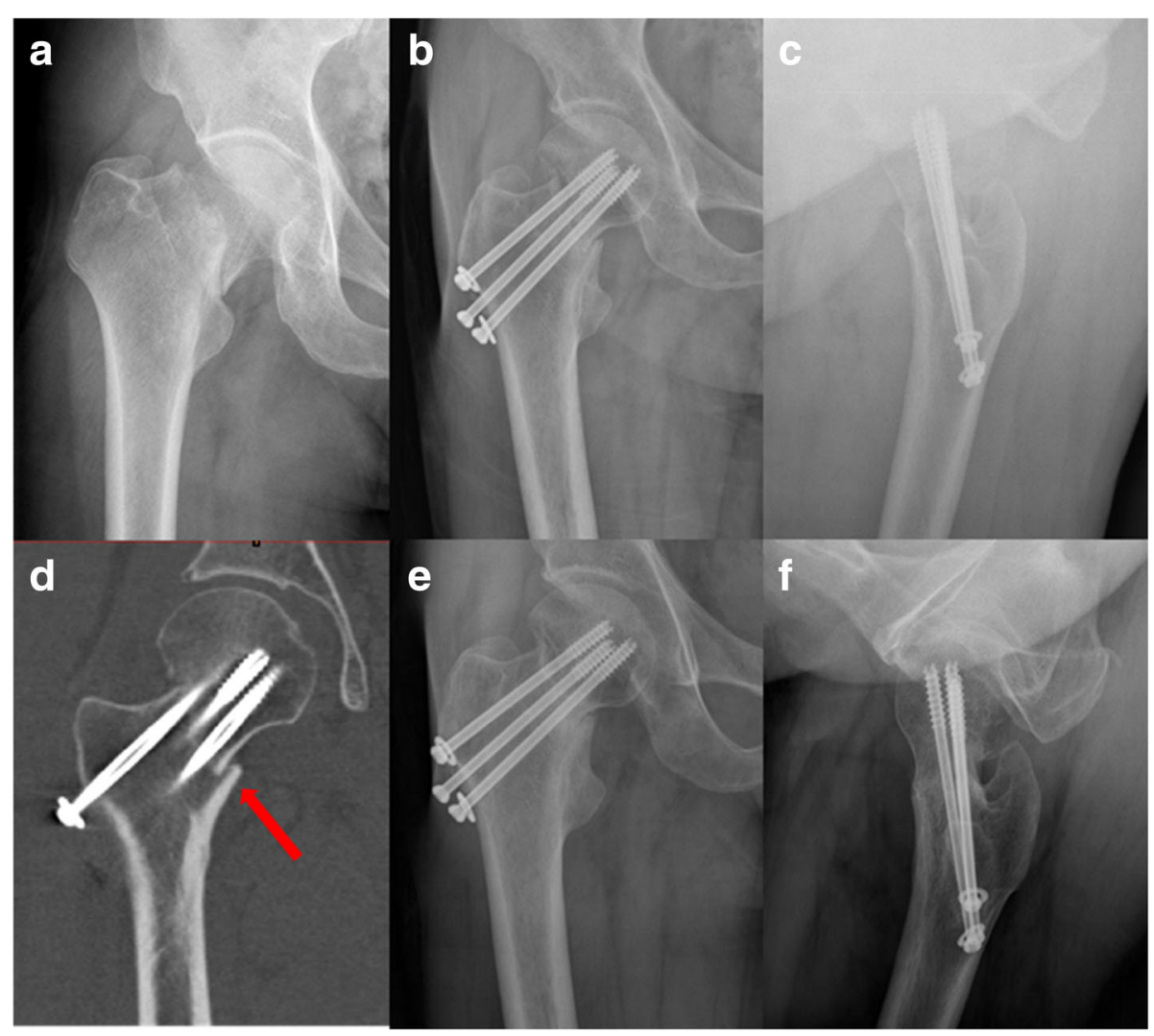

Fig. 4 AP view of femoral neck fracture in a 53-year-old male (a). The fracture was reduced with an inferior cortex support pattern (b, c). CT showed cortex buttress (red arrow, d). Although gradually displaced to varus $\left(>10^{\circ}\right)$ and shortening of the femoral neck, the fracture eventfully united and the patient restored full-weight-bearing walk in 6 months postoperatively $(\mathbf{e}, \mathbf{f})$

The mean shortening length of the femoral neck was $2.7 \mathrm{~mm}$ (Table 3). Using $5 \mathrm{~mm}$ as a criterion, three patients in group I had femoral neck shortening. One was diagnosed with avascular necrosis (AVN) 3 years postoperatively and accepted conversion to THA. The other two did not complain any discomfort with neck shortening.

\section{Discussion}

In the treatment of young adult femoral neck fractures, fracture initial displacement and the quality of reduction are the major determinant factors for outcomes. Anatomic reduction is the key to success in fracture union.
For grading the quality of femoral neck fracture reduction, two parameters are used: the degree of residual angulation (Garden alignment) and the amount of displacement (cortex apposition, regardless of direction). Excellent is defined as $<2 \mathrm{~mm}$ of displacement and $<5^{\circ}$ of angulation in any plane, good as 2 to $5 \mathrm{~mm}$ of displacement and $/$ or $5^{\circ}$ to $10^{\circ}$ of angulation, fair as $>5$ to $10 \mathrm{~mm}$ of displacement and/or $>10^{\circ}$ to $20^{\circ}$ of angulation, and poor as $>10 \mathrm{~mm}$ of displacement and/or $>20^{\circ}$ of angulation [7]. Based on the above criteria, Haidukewych et al. [7] reported that even with good to excellent fracture reduction in 46 patients, $11(24 \%)$ were associated with the development of osteonecrosis and $2(4 \%)$ were

Table 1 Demographic data of patients

\begin{tabular}{lllll}
\hline Item & Total $(n=46)$ & Group 1 $(n=30)$ & Group 2 $(n=16)$ & $p$ \\
\hline Male/female & $20 / 26$ & $13 / 17$ & $7 / 9$ & 0.610 \\
Age, years & $50.3 \pm 9.6$ & $49.2 \pm 11.0$ & $52.4 \pm 5.9$ & 0.594 \\
BMI & $22.9 \pm 1.6$ & $22.6 \pm 1.7$ & $23.5 \pm 1.2$ & 0.074 \\
ASA score & $1.3 \pm 0.5$ & $1.3 \pm 0.5$ & $1.4 \pm 0.5$ & 0.609 \\
FU time, months & $22.0 \pm 10.4$ & $22.9 \pm 11.7$ & $20.1 \pm 7.2$ & 0.871 \\
\hline
\end{tabular}

Note: Group $1=$ anatomic reduction group. Group $2=$ Gotfried reduction group. Data are expressed as mean \pm SD $B M I$ body mass index, ASA American Society of Anesthesiologists, FU follow-up 
Table 2 Comparison of complications of the two groups

\begin{tabular}{llll}
\hline Item & Group 1 $(n=30)$ & Group 2 $(n=16)$ & $p$ \\
\hline Complications & $6(20 \%)$ & $1(6.3 \%)$ & $1(6.3 \%)$ \\
$\quad$ Displacement to varus $\left(>10^{\circ}\right)$ & $3(10 \%)$ & $1(6.3 \%)$ & $/$ \\
Shortening $(>5 \mathrm{~mm})$ & $3(10 \%)$ & 0 & $/$ \\
Nonunion & $2(6.7 \%)$ & 0 & $/$ \\
Early failure & $1(3.3 \%)$ & 0 & $/$ \\
AVN & $1(3.3 \%)$ & 0 & 0.168 \\
Conversion to prosthetic replacement & $4(13.3 \%)$ & & \\
\hline
\end{tabular}

associated with the development of nonunion. Gardner et al. [8] noted that the rate of complication was $10 \%$ with "excellent" reduction quality, and 26\% with "good" reduction quality. Liporace et al. [9] reported that 8 of the $59(14 \%)$ vertical femoral neck fractures with good to excellent reduction quality had fracture nonunion.

However, the so called anatomic reduction in intraoperative fluoroscopic monitoring does not guarantee good outcomes postoperatively. During healing of femoral neck fracture, both bone resorption and shear forces of the fracture site can result in secondary sliding and displacement, which tend to cause femoral neck shortening and neck-shaft angle varus, even though the fracture was reduced in anatomic reduction quality [5]. It is well known that the varus displacement is a paramount predictor for failure in femoral neck fractures [10], and femoral neck shortening will decrease the purchase of screws and can also lead to failure [3]. Bone resorption is inevitable during healing of femoral neck fracture, and shear forces can hardly be controlled except for limited weight bearing. Therefore, techniques avoiding the negative affect of bone resorption and shear forces are reasonable to reduce the incidence of complications.

We think the so called anatomic cortical reduction shown in intraoperative fluoroscopy may actually contain three sub-conditions: some are exact anatomic cortical reduction, others slightly negative cortical reduction, and still others slightly positive reduction. But as the image resolution of intraoperative fluoroscopy was limited, those three sub-conditions were hard to be distinguished from one another. Negative cortical apposition means no cortical support and the head-neck fragment has a clear tendency to displace in a varus position [11]. After bone resorption of the fracture site, a slightly varus apposition may become truly varus and initiate displacement to cause fixation failure (Fig. 2) or fracture nonunion. This may explain the complications in our group I. We suggest that even though anatomic reduction is seen in intraoperative fluoroscopies, immediate postoperative radiographs and close postoperative follow-up are required to detect possible reduction changes. Intraoperative CT scanning is accurate to detect reduction quality, but it is not applicable in most hospitals.

Gotfried introduced the cortical buttress reduction, which was a non-anatomic functional reduction pattern [4]. The proximal head-neck fragment is slightly displaced superiorly to the distal fragment intentionally (less than one cortex thickness or $4 \mathrm{~mm}$ ), so that the head-neck is supported and is upheld by the inferior calcar cortex. And simultaneously, the head-neck fragment is usually located in a slight valgus position. After screw fixation, the femoral head-neck is dual buttressed by the fixation screw and the calcar cortex, and a sustainable fracture stability is restored.

In a Gotfried reduction pattern, the head-neck fragment was further supported by the inferior femoral neck cortical calcar, unlike in an anatomic reduction pattern, in which the head-neck fragment was only supported by the fixation screw. It can resist the vertical shear forces by inferior cortical contact, and transfer the shear forces to compress the bone substance of the fracture site, providing both mechanical and biological environment for the fracture stability and healing. Even though the

Table 3 Comparison of radiological assessment in the two groups

\begin{tabular}{|c|c|c|c|c|}
\hline Parameters & Total $(n=46)$ & Group $1(n=30)$ & Group $2(n=16)$ & $p$ \\
\hline Garden alignment AP angle & $165.1 \pm 3.3$ & $164.5 \pm 3.6$ & $166.2 \pm 2.5$ & 0.104 \\
\hline Garden alignment lateral angle & $178.2 \pm 1.5$ & $178.2 \pm 1.5$ & $178.0 \pm 1.5$ & 0.714 \\
\hline Varus change of the FNSA, degrees & $3.4 \pm 3.9$ & $3.5 \pm 4.2$ & $3.1 \pm 3.4$ & 0.715 \\
\hline Shortening length of the femoral neck, mm & $3.0 \pm 2.8$ & $3.3 \pm 3.4$ & $2.6 \pm 1.3$ & 0.914 \\
\hline
\end{tabular}

Note: Garden alignment index angles (AP and lateral views) were measured on intraoperative fluoroscopy FNSA femoral neck-shaft angle 
fracture displaced to varus because of bone resorption, fracture union can be anticipated because of the compression of the fracture site (Fig. 4), i.e., an inferior cortical buttress reduction pattern can reduce the negative affect of fracture site resorption. In our group II of 16 patients, fracture union was eventfully achieved in all cases, with only one patient who developed hip varus.

In 2015, Mir and Collinge hypothesized the application of a small medial buttress plate to augment fixation in vertical shear femoral neck fractures [12]. In 2017, Ye et al. [13] reported the preliminary clinical results in 28 cases of vertical femoral neck fractures treated with open reduction and three cannulated screws and augmented with a medial buttress plate. Despite all fracture reduction achieved Garden's alignment index grade 1, reduction loss with backing out of the cannulated screws was found in three cases, with plate screws broken in one case in 3 months of follow-up. The medial buttress plate fixation can effectively resist shearing force of the fracture site to avoid displacement to varus; however, it cannot prevent the negative affect of bone resorption and needs additional exposure.

There are several advantages of an inferior cortical buttress reduction pattern. It emphasizes the direction of cortical apposition, using the inferior calcar cortex to uphold the head-neck fragment. It is a non-anatomic pattern and is easy to achieve by closed maneuver, and no need of additional operation. It has favorable biomechanical properties after fracture site resorption and sliding. Our preliminary results show that it is an effective clinical alternative to the anatomic reduction.

Some limitations of the present retrospective study must be acknowledged. Firstly, it is a preliminary report with a small number of cases. Secondly, we found no case of AVN in group II. It may be due to the short follow-up period. Larger-scale and long-term follow-up studies are needed to confirm the ultimate outcome with a Gotfried non-anatomic cortical buttress reduction pattern.

\section{Conclusion}

Our preliminary study showed that fracture reduction with an inferior cortical buttress pattern, though non-anatomic, can produce excellent outcomes in closed fixation of displaced femoral neck fractures. Both calcar support reduction and anatomic reduction are acceptable in clinical practice.

\section{Abbreviations \\ ASA: American Society of Anesthesiologists; BMI: Body mass index; FNL: Femoral neck length; FNSA: Femoral neck-shaft angle; FU: Follow-up; THA: Total hip arthroplasty}

\section{Funding}

The study was supported by National Natural Science Foundation of China (NSFC, No. 81772323) and Science and Technology Commission of Shanghai Municipality, China (No. 14411971900).

\section{Availability of data and materials}

All data used and analyzed during this study are available from the corresponding author on reasonable request.

\section{Authors' contributions}

WFX and SMC performed the study design, analyzed the results, and contributed to the manuscript. YQZ made some meaningful suggestions.

$\mathrm{SJH}$ and SCD contributed to the collection of the cases. All authors reviewed and approved the final submitted version.

Ethics approval and consent to participate

The study was approved by Institutional Review Board of Yangpu Hospital, Tongji University (No. 2017ZRKX-013). Written informed consent was obtained from all patients.

Consent for publication

Not applicable.

\section{Competing interests}

The authors declare that they have no competing interests.

\section{Publisher's Note}

Springer Nature remains neutral with regard to jurisdictional claims in published maps and institutional affiliations.

\section{Author details}

${ }^{1}$ The Department of Orthopaedic Surgery, Yangpu Hospital, Tongji University School of Medicine, 450Tengyue Road, Shanghai 200090, People's Republic of China. ${ }^{2}$ The Department of Orthopaedic Surgery, Tongji Hospital, Tongji University School of Medicine, Shanghai 200090, People's Republic of China.

Received: 16 October 2018 Accepted: 21 February 2019

Published online: 28 February 2019

References

1. Huang TW, Hsu WH, Peng KT, Lee CY. Effect of integrity of the posterior cortex in displaced femoral neck fractures on outcome after surgical fixation in young adults. Injury-international Journal of the Care of the Injured. 2011; 42:217-22. https://doi.org/10.1016/j.injury.2010.10.005.

2. Yang JJ, Lin LC, Chao KH, Chuang SY, Wu CC, Yeh T, et al. Risk factors for nonunion in patients with intracapsular femoral neck fractures treated with three cannulated screws placed in either a triangle or an inverted triangle configuration. J Bone Joint Surg Am. 2013;95:61-9. https://doi.org/10.2106/ JBJS.K.01081.

3. Chua D, Jaglal SB, Schatzker J. Predictors of early failure of fixation in the treatment of displaced subcapital hip fractures. J Orthop Trauma. 1998;12: 230-4.

4. Gotfried Y. The Gotfried (nonanatomic, closed) reduction of unstable subcapital femoral fractures. Tech Orthop. 2012;27:259-61. https://doi.org/ 10.1097/BTO.0b013e318263f2f8.

5. Gotfried Y, Kovalenko S, Fuchs D. Nonanatomical reduction of displaced subcapital femoral fractures (Gotfried reduction). J Orthop Trauma. 2013;27: e254-9. https://doi.org/10.1097/BOT.0b013e31828f8ffc.

6. Zlowodzki M, Ayieni O, Petrisor BA, Bhandari M. Femoral neck shortening after fracture fixation with multiple cancellous screws: incidence and effect on function. J Trauma. 2008;64:163-9. https://doi.org/10.1097/01.ta. 0000241143.71274 .63 .

7. Haidukewych GJ, Rothwell WS, Jacofsky DJ, Torchia ME, Berry DJ. Operative treatment of femoral neck fractures in patients between the ages of fifteen and fifty years. J Bone Joint Surg (Am Vol). 2004;86-A:1711-6.

8. Gardner S, Weaver MJ, Jerabek S, Rodriguez E, Vrahas M, Harris M. Predictors of early failure in young patients with displaced femoral neck fractures. J Orthop. 2015;12:75-80. https://doi.org/10.1016/j.jor.2014.01.001.

9. Liporace F, Gaines R, Collinge C, Haidukewych GJ. Results of internal fixation of pauwels type-3 vertical femoral neck fractures. Journal of Bone \& Joint Surgery-american Volume. 2008;90:1654-9. https://doi.org/10.2106/JBJS.G. 01353.

10. Krischak G, Beck A, Wachter N, Jakob R, Kinzl L, Suger G. Relevance of primary reduction for the clinical outcome of femoral neck fractures treated with cancellous screws. Arch Orthop Trauma Surg. 2003:123:404-9. https:// doi.org/10.1007/s00402-003-0571-3. 
11. Zhang YQ, Chang SM. Mechanism of "Gotfried reduction" in femoral neck fracture. J Orthop Trauma. 2013;27:e291. https://doi.org/10.1097/BOT. 0000000000000007.

12. Mir $\mathrm{H}$, Collinge $\mathrm{C}$. Application of a medial buttress plate may prevent many treatment failures seen after fixation of vertical femoral neck fractures in young adults. Med Hypotheses. 2015;84:429-33. https://doi.org/10.1016/j. mehy.2015.01.029.

13. Ye Y, Chen K, Tian K, Li W, Mauffrey C, Hak DJ. Medial buttress plate augmentation of cannulated screw fixation in vertically unstable femoral neck fractures: surgical technique and preliminary results. Injury. 2017;48: 2189-93. https://doi.org/10.1016/j.injury.2017.08.017.

Ready to submit your research? Choose BMC and benefit from:

- fast, convenient online submission

- thorough peer review by experienced researchers in your field

- rapid publication on acceptance

- support for research data, including large and complex data types

- gold Open Access which fosters wider collaboration and increased citations

- maximum visibility for your research: over $100 \mathrm{M}$ website views per year

At BMC, research is always in progress.

Learn more biomedcentral.com/submissions 